\title{
Analysis of the surface of healthy and fluorotic human enamel using microhardness test
}

\author{
A. Monjaras-Avila, V. Zavala-Alonso*, G. Morales-Alcocer, G.A. Martinez-Castanon, \\ N. Patiño-Marin, J. Ramirez-Gonzalez \\ Programa Doctoral en Ciencias Odontológicas, Facultad de Estomatología, Universidad Autónoma de San Luis Potosí \\ Dr. Manuel Nava 2, Zona Universitaria, San Luis Potosí, SLP, 78290, México.
}

(Received: January 17th, 2016; Accepted: March 1st, 2017)

\begin{abstract}
The microhardness is an essential property of tooth enamel; there may be many factors that alter or diminish this quality causing weakness, one of which is dental fluorosis. The aim of this study was to evaluate the surface microhardness of fluorotic enamel compared with healthy enamel. Two hundred forty extracted human molars were classified into four groups: Healthy $(\mathrm{H})$, mild (MI), moderate (MO) and severe (S) fluorosis according to the Dean index. Micro Vickers Hardness Tester was used to analyze all samples. Average, standard deviation and ranges were calculated for quantitative variables, the ANOVA and Tukey test was used to identify differences between groups. The mean values of surface microhardness in HVN were: H, 333.4; MI, 290.3; MO, 266.1; S, 252.0. The differences between mean surface microhardness among healthy group and fluorotic groups were statistically significant $(\mathrm{p}<0.05)$. This in vitro study confirms that surface microhardness decreased according to the severity of fluorosis.
\end{abstract}

\section{Introduction}

Dental fluorosis is a tooth malformation that is caused by chronic ingestion of high levels of fluoride $(\mathrm{F})$ during tooth development [1]. In addition, delayed removal of the enamel matrix proteins may play a role in the hypo mineralization defects seen in fluorosed enamel. Most of these proteins are absent in the mature tissue of these moderately fluorosed teeth [2].

Fluorosis has increased throughout the world, [3] ranging from $7.7 \%$ to $80.9 \%$ in areas with fluoridated water and from $2.9 \%$ to $42 \%$ in areas without fluoridated water [4-7]. In San Luis Potosí, Mexico, the fluorosis prevalence was $69 \%$ where the levels of water fluorine were less than $0.7 \mathrm{ppm}$, and increased to $98 \%$ for a fluorine level of $2 \mathrm{ppm}$ [8]. Fluorosis is likely the best studied and possibly has the most adverse effect of any agent on the formation of enamel. However, little information exists. According Vieira quality tooth refers to the ability of the tooth to perform its function, maintenance of masticatory forces, and can be evaluated by measuring a tooth material, mechanical and structural properties [9]. The present authors have reported information about fluorotic enamel structure, our studies confirm at the nanometer level that there is a positive association between fluorosis severity, enamel surface roughness and absolute depth profile and there is an association with the clinical findings of fluorosis measured by fluorosis indexes [10]. However, information on the mechanical properties of the fluorotic enamel is sparse. Hardness is regarded as an essential physiological property of the enamel, a result of the interaction of numerous properties such as strength, ductility, malleability and resistance to abrasion and cutting [11]. Microhardness has since proven to be a sufficiently sensitive test when it comes to superficial lesions because it can detect early stages of demineralization. Therefore, both microhardness analysis and the analysis of surface topography are quality indicators of tooth enamel to resist erosion processes [12-14]. The microhardness of the enamel is determined using Vickers microhardness testing.

The main purpose of this study was to obtain the surface microhardness of the fluorotic enamel compared with healthy control enamel.

\section{Materials and methods}

\section{Subjects and Sample Preparation}

Patients undergoing extraction of third molars at hospital and private clinics were asked to donate their extracted teeth, and then informed patient consent was obtained. Erupted third molars were collected from three different locations: (1) Ciudad Valles (San Luis Potosí, México), which has a water fluoride level between 0.1 and 0.6 ppm F; (2) San Luis Potosi City (México) with a natural fluoride level between 0.7 and 2 ppm F; and (3) Salitral de Carrera (San Luis Potosí, México) with a natural fluoride level between 2 and 5 ppm. All samples were cleaned and disinfected in an ultrasonic bath (Biosonic UC300-115B, Colténe/Whaladent, Cuyahoga Falls, Ohio,USA), then washed in running water, dried, an analyzed by visual observation for fluorosis severity according to the Dean index [15]. The study was blinded for the clinical diagnosis of dental fluorosis; a second observer carried out Microhardness evaluation.

The selected molars were divided into four groups of 60 samples each: the Healthy group $(\mathrm{H})$, the Mild group (MI), the Moderate group (MO), and the Severe group (S). All molars were stored in distilled water (Milli-Q, Millipore Co., Billerica, MA, USA) at $4{ }^{\circ} \mathrm{C}$ until experimental procedures were performed. Each buccal surface of molars healthy and pathologic fluorosis was sectioned perpendicular to the long axis of the tooth by means of a water-cooled low-speed diamond saw (\#7910, medium size grain; Brasseler, Savannah, GA, USA) to obtain samples $3 \mathrm{~mm}$ in width (Figure 1A). The samples were then mounted in acrylic blocks (Figure 1B), followed by ultrasonic cleansing in distilled water.

\section{Microhardness test}

All samples were subjected to hardness indentations made with the Vickers hardness machine HV-1000 (DongGuan Sinowon Precision Instrument Co., Ltd. South District DongGuan, China) using a $50 \mathrm{gf}$ load and a dwell time of 30 seconds. Three indentations separated by $0.4 \mathrm{~mm}$, were made 
Table 1. Surface Microhardness (HVN) in all study groups.

\begin{tabular}{lcc}
\hline \multirow{2}{*}{ Group } & \multicolumn{2}{c}{$\begin{array}{c}\text { Microhardness } \\
\text { (HVN) }\end{array}$} \\
\cline { 2 - 3 } & Mean \pm SD & Range \\
\hline Healthy & $333.4 \pm 45$ & $443.07-211.2$ \\
Mild & $290.3 \pm 63$ & $511.93-162.67$ \\
Moderate & $266.1 \pm 61$ & $467.5-119.2$ \\
Severe & $252.0 \pm 70$ & $464.9-115.7$ \\
\hline $\mathrm{n}=60$ samples per group &
\end{tabular}

for each sample and the average value was recorded as the surface microhardness.

\section{Statistical Analysis}

Examiners were calibrated with an expert in fluorosis by using the intraclass correlation coefficient (ICC). All data are expressed as mean value \pm standard deviation and range. Shapiro-Wilks and Brown Forsythe tests were used to assess the normality of the data distribution. One-way analysis of variance (ANOVA) and Tukey's multiple comparison tests were used to compare microhardness among groups. The JMP program (version 9) and Stat View (both from SAS Institute, Cary, NC, USA) were used for statistical analysis, and $\mathrm{p}<0.05$ was considered statistically significant.

\section{Results}

The interobserver reproducibility analysis fluorosis achieved by the examiner and the expert revealed an ICC of 0.99. The distribution of all variables was parametric. 240 samples were obtained, 60 in each group. The microhardness mean values, the standard deviation, and the ranges of the total number of indentations for each group are shown in Table 1. The mean microhardness and standard deviation in HVN were: Healthy Group, $333.4 \pm 45$ HVN; Mild Group, $290.3 \pm 63$ HVN; Moderate Group 266.1 \pm 61 HVN and Severe group $252.0 \pm 70 \mathrm{HVN}$. In Table 2, the Tukey test showed that differences between mean microhardness for most variables were statistically significant $(p<0.05)$ among groups, but there was no difference between Groups Mild versus Moderate and Moderate versus Severe ( $p>0.05$ ). Figure 2 shows representative images of the Vickers indentations from the different groups.
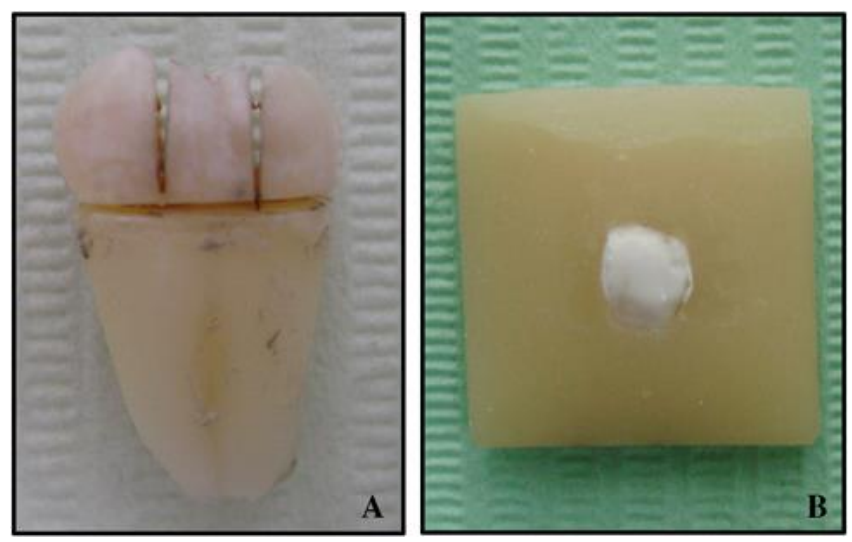

Figure 1. A: Tooth was sectioned on the buccal face to obtain $3 \mathrm{~mm}$ width samples. B: Sample was mounted in acrylic blocks.
Table 2. Surface microhardness $p$ values comparisons between groups.

\begin{tabular}{cc}
\hline Groups $^{\text {a }}$ & $\boldsymbol{P}$ \\
\hline H versus MI & 0.0007 \\
H versus MO & 0.0001 \\
H versus S & 0.0001 \\
MI versus MO & 0.1283 \\
MI versus S & 0.0035 \\
MO versus S & 0.5795 \\
${ }^{\text {a }}$ H, Healthy group; MI, mild fluorosis group; MO, \\
moderate fluorosis group; $S$, severe fluorosis group.
\end{tabular}

\section{Discussion}

Although a large number of epidemiological studies of fluorosis have been reported, and the present authors have previously reported molecular structure, roughness, and absolute depth profile of fluorotic enamel compared with healthy enamel, to our knowledge this is the first study to compare the surface microhardness of healthy and fluorotic human enamel. There is evidence that $\mathrm{F}$ content is higher in enamel when the time from completion of enamel formation is extended before eruption occurs, such as is the case of third molars, which start mineralization relatively late and commonly stay unerupted for long periods [16]. On average, a period of 6 years can be expected between completion of enamel formation and tooth eruption, as observed in premolars, teeth that have been reported are the most affected by dental fluorosis [14]. Erupted third molars were used because it has been reported that they exhibit higher degrees of dental fluorosis than unerupted teeth $[17,18]$. The fluorosis diagnostic was performed using the Dean index. This form became the most universally accepted classification system for dental fluorosis. An individual's fluorosis score is based on the most severe form of fluorosis found on two or more teeth [15]. It has been reported that fluorosis is a developmental enamel disturbance caused by sustained exposure to high concentrations of fluoride during tooth development, leaving to enamel with a lower mineral content because changes in the structure of external surfaces [19]. Static methods by Knoop and Vickers are used to measure microhardness of hard dental tissues. Knoop's test for microhardness has been adopted as one of the main experimental methods for the analysis of changes in enamel a dentin physical properties after exposures to various treatments [20]. In this study we determined the surface microhardness of healthy human teeth and affected by different degrees of fluorosis, with the method of microhardness Vickers, as it is most appropriate to compare variations of the mechanical properties of an anisotropic material as is tooth enamel and which has not been exposed to any treatment [20], also, Vickers indentations are influenced less by specimen surface flatness, parallelism, and surface finish than Knoop indentations [21], Sides being a simple and efficient method as a noninvasive technique allowing the use of the sample in more than one occasion. The microhardness of enamel varies in different areas of the same tooth; therefore, any individual measurement may not accurately reflect the overall microhardness of dental enamel [22]. Therefore, in this study, measurements were performed 

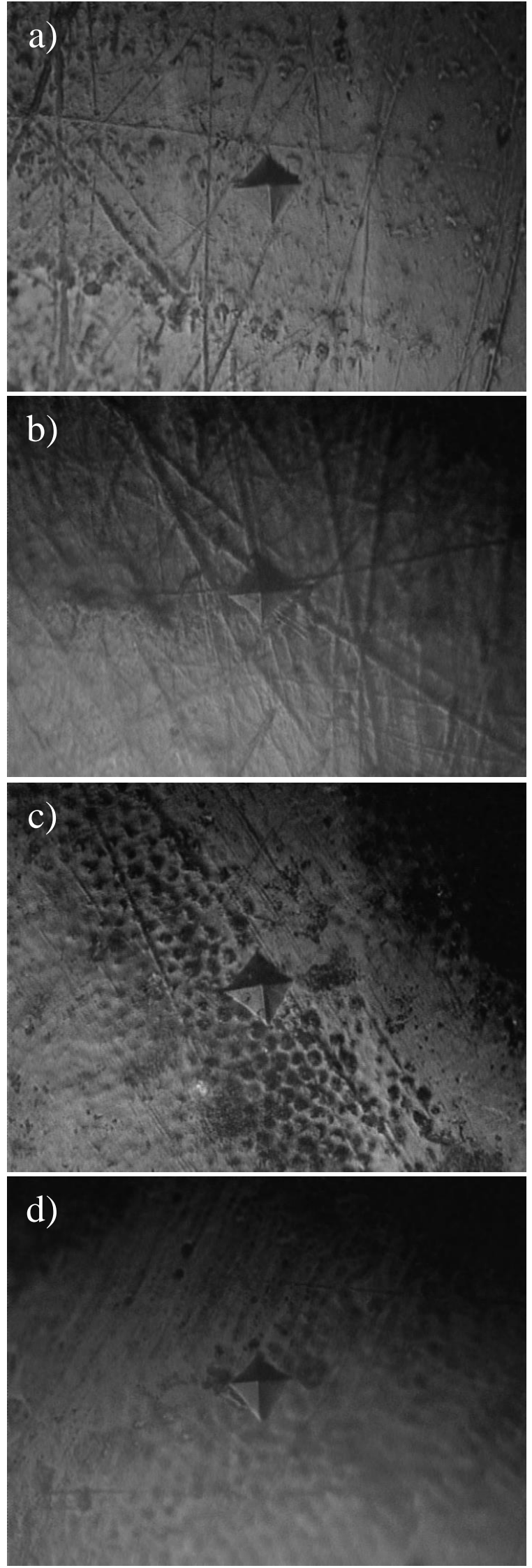

Figure 2. Representative images of the Vickers indentations (Vickers Measurement Software iVicky V2.0, Sinowon) of the different study groups: a) Healthy; b) Mild; c) Moderate; d) Severe. in triplicate in different areas of the enamel, and then a mean was obtained for each sample. Research on enamel microhardness is sparse, the number of samples is always reduced, and have various methodologies in relation to the load used [23]. In the literature review was founded that there is no standard as to the loads and time used [17]. We decided to use the load of 50 grams for 30 seconds because the indentations appeared clearest diagonal edges, geometric and free of irregularities in the test area, as well as minor deformity that increase or decrease the load, since these variables may influence the actual results of hardness. It was decided to perform the measurement of hardness in the outermost of the enamel, since, according Gutierrez Salazar [22]. There is no statistically significant difference in Vickers hardness values of the external surface of the enamel to the enamel-dentin junction as these values remain constant throughout the thickness of the enamel, also adds that the larger hardness is only along the cross section of the longitudinal section. To our knowledge, this is the first time that microhardness test has been used as indicator of the mechanical properties of the enamel surface by analyzing healthy enamel in a gradient of fluorosis severity. Microhardness was negatively correlated with increased fluorosis severity $(\mathrm{H}, 333.4 \mathrm{HVN}$; MI, $290.3 \mathrm{HVN}$; MO, 266.1 HVN; S, 252 HVN). Differences between the mean microhardness of all fluorosis groups with healthy group were statistically significant $(\mathrm{p}<0.05)$. The mean microhardness of enamel surfaces showed the highest values for Group $\mathrm{H}$ and lowest values for Group S, so it is clear, that microhardness decreased according to the severity of fluorosis. This investigation showed an inverse relationship between fluorosis severity and surface microhardness, this means that a greater degree of enamel fluorosis is less degree of hardness. These findings could show that dental fluorosis negatively affects the process of mineralization of the enamel outermost layer and their mechanical properties although we agree with Vieira et al. 2004 on the fact that other factors, such as individual genetic variation, can play an important role in DF severity and its consequences. Another study by our research group showed a positive association between fluorosis severity and enamel surface roughness (ESR) and absolute depth profile (ADP), finding that the greater severity of fluorosis increased enamel surface roughness and depth profile values [10].

The mean value of $333.4 \pm 45 \mathrm{HVN}$ in healthy enamel, were similar with obtained by the study previously published by S. Hayashi-Sakai in 2012 where obtained values of $319 \pm 28.3$ HVN however, Aylin Sakar-Deliormanli and Mustafa Güden in 2006 obtained mean values of 283.1 HVN which resemble our values obtained with mild fluorosis enamel $290.3 \pm 63 \mathrm{HVN}$, but both with loads well above that of our study ranging from 200 to $500 \mathrm{gf}[24,25]$.

In the in vitro study by Priyadarshini in 2013, the effect of topical application of the organic fluoride in comparison inorganic fluoride in enamel microhardness was evaluated, [26] hardness values reported in healthy enamel were of 448.70 HVN, increasing values with inorganic fluoride 460.43 - 461.49 HVN and 474.82 HVN with organic fluoride, observing higher values when compared to our 
study, because topical application of this has an important role in enamel remineralization, acting as a catalyst and influencing reaction rates dissolution and processing of various calcium phosphate mineral, action does not happen when fluoride acts systemically, as in dental fluorosis. On the other hand in a study by Vieira et al. 2005 made quantitative fluorescence induced in mice, the severity of dental fluorosis and enamel microhardness was evaluated [9], resulting in the following data; in water with $0 \mathrm{ppm}$ fluoride averaged $160.3 \mathrm{HVN}$; 25 ppm: $145.5 \mathrm{HVN}$; 50 ppm: 143 HVN; 100 ppm: 118.7 HVN; showing a decrease in hardness values with increasing amount of fluoride ppm in water, observing the same trend as in our research, but unlike our study, mice were subjected to fluoride levels for six weeks having a control intake, and also the severity of the disease is more severe in this type of sample, as well as enamel specimens were polished in our study and measurement of samples was performed intact.

The importance of this study in human dental enamel affected by fluorosis knows one of the structural and mechanical properties of the biggest teeth and also is considered an essential physiological property, which is affected by this disease, combining this the development of caries [27], and tendency to fracture. Therefore, it is important to determine the influence of fluoride on microhardness and mineralization of the teeth, both of which affect the quality of the dental organs.

\section{Conclusions}

It was concluded that the severity of dental fluorosis has influence on mechanical properties such as hardness of tooth enamel, being lower hardness values with increasing degree of dental fluorosis. The results could indicate the probable susceptibility of teeth affected by fluorosis to diseases such as dental caries.

\section{Acknowledgments}

This work was supported by Consejo Nacional de Ciencia y Tecnología (CONACYT CB-178261).

\section{References}

[1]. P. Den Besten, Adv. Dent. Res. 8, 105 (1994).

[2]. J. Wright, S. Chen, K. Hall, M. Yamauchi, J. Bawden, J. Dent. Res. 75, 1936 (1996).
[3]. B. Angmar Månsson, E. Jong, F. Sundstrom, J. Bosch, $\underline{A d v}$. Dent. Res. 8, 75 (1994).

[4]. D. Clark, Community Dent. Oral Epidemiol. 22,148 (1994).

[5]. A. Mascarenhas, Pediatr. Dent. 22, 269 (2000).

[6]. D. Pendrys, J. Am. Dent. Assoc. 131,746 (2000).

[7]. E. Everett , M. Mchenry, N. Reynolds, H. Eggertsson, J. Sullivan, C. Kantmann, E. Martínez Mier, J. Warrick, G. Stookey, J. Dent. Res. 81, 794 (2002).

[8]. V. Zavala Alonso, J. Loyola Rodríguez, H. Terrones, N. Patiño Marín, G. Martínez Castañón, K. Anusavice, J. Oral Sci. 54, 93 (2012).

[9]. A. Vieira, R. Hanocock, H. Eggertsson, E. Everett, M. Grynpas, Calcif. Tissue Int. 76, 17 (2005).

[10]. V. Zavala Alonso, G. Martínez Castañón, N. Patiño Marín, H. Terrones, K. Anusavice, J. Loyola Rodriguez, Microsc. Microanal. 16, $531(2010)$.

[11]. C. Davidson, E. Hoekstra, J. Arends, Caries Res. 144, 135 (1974).

[12]. E. Correa Olaya, M. Mattos Vela, Kiru 8, 88 (2011).

[13]. M. Medeiros, H. Carlo, R. Lacerda-Santos, B. Lima, F. Souza, J. Rodrigues, F. Carvalho, Braz. Oral Res. 30, 1 (2016).

[14]. S. Kwek, M. Mian, C. Hall, Z. Xie, R. Yong, J. Kaidonis, G. Townsend, S. Ranjitkar, Australian Dental Journal 60, 12 ( 2015). [15]. H. Dean, R. Dixon, C. Cohen, Public Health Reports (18961970) 50, 424 (1935).

[16]. A. Vieira, R. Hancock, H. Limeback, R. Maia, M. Grynpas, $\underline{J}$. Dent. Res. 83, 76 (2004).

[17]. A. Richards, S. Likimani, V. Baelum, O. Fejerskov, $\underline{\text { Caries }}$ Res. 26, 328 (1992).

[18]. O. Fejerskov, M. Larsen, A. Richards, V. Baelum, Adv. Dent. Res. 8, 15 (1994).

[19]. J. Abanto Alvarez, K. Rezende, S. Salazar Marocho, F. Alves, P. Celiberti, A. Ciamponi, Med. Oral Patol. Oral Cir. Bucal 1, 103 (2009).

[20]. Y. Zhang, W. Du, X. Zhou, H. Yu, Int. J. Oral Sci. 6, 61 (2014).

[21]. ASTM C1327-08, Standard Test Method for Vickers Indentation Hardness of Advanced Ceramics (ASTM International, West Conshohocken, PA, 2008).

[22]. M. Gutiérrez Salazar, J. Reyes Gasga, Materials Research 6, $367(2003)$.

[23]. R. Wang, Dent. Mater. 21, 429 (2005).

[24]. S. Hayashi Sakai, J. Sakai, M. Sakamoto, H. Endo, J. Mater. Sci. Mater. Med. 23, 2047 (2012).

[25]. A. Sakar-Deliormanli A, M. Güeden, J. Biomed. Mater. Res. B Appl. Biomater. 76, 257 (2006).

[26]. S. Priydarshini, R. Ramya, A. Shetty, P. Gautham, S. Reddy, R. Srinivasan, J. Conserv. Dent. 16, 203 (2013).

[27]. P. Waidyasekera, T. Nikaido, D. Weerasinghe, K. Wettasinghe, J. Tagami, J. Dent. 35, 343 (2007).

(C) 2017 by the authors; licensee SMCTSM, Mexico. This article is an open access article distributed under the terms and conditions of the Creative Commons Attribution license (http://creativecommons.org/licenses/by/4.0/). 